\title{
New Superhard Carbon Phases Between Graphite and Diamond
}

\author{
Chaoyu He, ${ }^{1}$ L. Z. Sun,, C. X. Zhang, ${ }^{1}$ K. W. Zhang, ${ }^{1}$ Xiangyang Peng, ${ }^{1}$ and Jianxin Zhong ${ }^{1,0}$ \\ ${ }^{1}$ Institute for Quantum Engineering and Micro-Nano Energy Technology, Xiangtan University, Xiangtan 411105, China
}

(Dated: October 29, 2018)

\begin{abstract}
Two new carbon allotropes (H-carbon and S-carbon) are proposed, as possible candidates for the intermediate superhard phases between graphite and diamond obtained in the process of cold compressing graphite, based on the results of first-principles calculations. Both H-carbon and S-carbon are more stable than previously proposed M-carbon and W-carbon and their bulk modulus are comparable to that of diamond. H-carbon is an indirect-band-gap semiconductor with a gap of $4.459 \mathrm{eV}$ and S-carbon is a direct-band-gap semiconductor with a gap of $4.343 \mathrm{eV}$. The transition pressure from cold compressing graphite is $10.08 \mathrm{GPa}$ and $5.93 \mathrm{Gpa}$ for H-carbon and S-carbon, respectively, which is in consistent with the recent experimental report.
\end{abstract}

PACS numbers: 61.50.Ks, 61.66.Bi, 62.50. -p, 63.20. D-

\section{INTRODUCTION}

Carbon is considered as the most active element in the periodic table due to its broad $\mathrm{sp}, \mathrm{sp}^{2}$ and $\mathrm{sp}^{3}$ hybridizing ability. Besides the four best-known carbon allotropes, graphite, cubic-diamond, hexagonal diamond and amorphous carbon, an unknown superhard phase

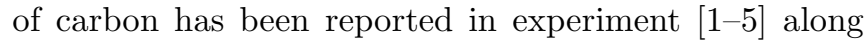
with the structural phase transition in cold compressed graphite. Several structures have been proposed as the candidates for this superhard phase, such as the monoclinic M-carbon [6, 7], cubic body center $\mathrm{C} 4$ carbon (bct-C4) 8] and the orthorhombic W-carbon [9]. The cohesive energy of the most stable $\mathrm{W}$-carbon is about $160 \mathrm{meV}$ per atom higher than that of diamond, indicating that other more stable carbon phases may exist. Very recently, another new carbon allotrope, named as Z-carbon, was proposed and investigated at almost the same time by three independent research groups $10-12]$. Z-carbon is more stable (its cohesive energy is about $129 \mathrm{meV}$ per atom larger than that of diamond) and harder than $\mathrm{W}$-carbon. Moreover, its transition pressure is around $10 \mathrm{Gpa}$ which is lower than that of W-carbon. Thus, it is believed that the formation in the process of the cold compressing graphite prefers Z-carbon rather than W-carbon. Although none of them can fit the experimental results satisfactorily, these theoretically proposed phases are significant in understanding the experimental process of cold compressing of graphite.

The formation of the new carbon phase needs large lattice distortions, such as buckling between adjacent carbon layers of graphite. Therefore, the phase transition depends not only on the particular nucleation history [13] but also on the structure of the starting graphitic material. Intuitively, such buckling between carbon layers will start from the natural $\mathrm{AB}$ stacking graphite rather than the AA stacking graphite chosen in previous theoretical studies 7 -12]. From this hypothesis, we propose two new superhard crystals, namely

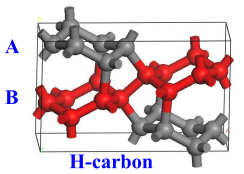

(a)

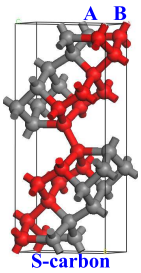

(d)

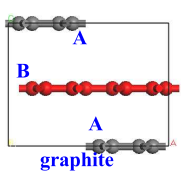

(b)

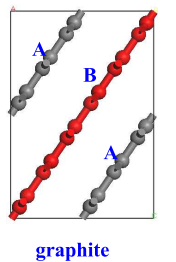

(e)
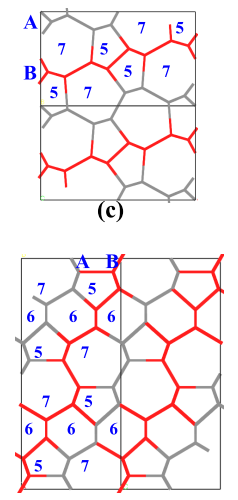

(f)
FIG. 1: Crystal structure of H-carbon(a), initial AB stacking graphite supercell for $\mathrm{H}$-carbon (b) and side view containing five and seven carbon rings of $\mathrm{H}$-carbon (c). Crystal structure of S-carbon (d), initial AB stacking graphite supercell for Scarbon (e) and side view containing five and seven carbon rings of S-carbon (f).

$\mathrm{H}$-carbon and S-carbon, as potential candidates for the experimentally observed intermediate state of carbon, which can be formed from compressing $\mathrm{AB}$ stacking graphite. Our first-principles calculations based on density functional theory show that both the allotropes are transparent wide band gap insulators and their hardness are comparable to that of diamond. S-carbon has the lowest enthalpy compared with bct-C4 carbon, $\mathrm{M}$-carbon, $\mathrm{W}$-carbon, and Z-carbon and is the most stable new carbon phase theoretically proposed until now. H-carbon is always more stable than $\mathrm{W}$-carbon and M-carbon. When the pressure is above $5.93 \mathrm{Gpa}$ (10.08 Gpa), S-carbon (H-carbon) is more favorable than graphite. 


\section{MODELS AND METHODS}

$\mathrm{H}$-carbon and S-carbon belong to the orthorhombic lattice and they can be obtained from cold compressing $\mathrm{AB}$ stacking graphite. Crystal structures of H-carbon and S-carbon are shown in Fig. 1 (a) and (d), and their corresponding initial graphite structures are shown in Fig. 1(b) and (e), respectively. H-carbon with the Pbam symmetry containing 16 carbon atoms per crystal cell is obtained by reorganizing $\mathrm{C}$ atoms to form $\mathrm{sp}^{3}$ bonds through compressing an $\mathrm{AB}$ stacking graphite supercell with 16 atoms. While, S-carbon with Cmcm symmetry containing 24 carbon atoms per crystal cell is obtained from larger $\mathrm{AB}$ stacking graphite supercell with 24 atoms.

All calculations are carried out using the density functional theory within both local density approximation (LDA) 14, 15] and general gradient approximation (GGA) [16] as implemented in Vienna ab initio simulation package (VASP) [17, 18]. In evaluating of the transition pressure from graphite to each superhard phases, it is known that LDA is a better choice because LDA can give reasonable interlayer distances, mechanical properties of graphite sheets due to a delicate error cancelation between exchange and correlation in comparison with that of semi-local generalized gradient approximation (GGA). The interactions between nucleus and the $2 \mathrm{~s}^{2} 2 \mathrm{p}^{2}$ valence electrons of carbon are described by the projector augmented wave (PAW) method [19, 20]. A plane-wave basis with a cutoff energy of $500 \mathrm{eV}$ is used to expand the wave functions. The Brillouin Zone (BZ) sample meshes are set to be dense enough to ensure the accuracy of our calculations 21]. Crystal lattices and atom positions of graphite, diamond, M-carbon, $\mathrm{W}$ carbon, Z-carbon, H-carbon and S-carbon are fully optimized up to the residual force on every atom less than $0.005 \mathrm{eV} / \AA$ through the conjugate-gradient algorithm. Vibration properties are calculated by using the phonon package [22] with the forces calculated from VASP.

\section{RESULTS AND DISCUSSION}

Similar to M-carbon and W-carbon, H-carbon and S-carbon contain distorted five and seven carbon rings, as shown in Fig. 11 (c) and (f). Their difference from $\mathrm{Z}$-carbon and bct-C4 carbon is the absence of four and eight carbon rings. The crystal structure of H-carbon belongs to the Pbam space group. At zero pressure, the GGA calculated equilibrium lattice constants are $\mathrm{a}=7.874 \AA, \mathrm{b}=4.807 \AA$ and $\mathrm{c}=2.524 \AA$. Four inequivalent atoms in this crystal occupy the positions at $(0.046$, $0.361,0.500), \quad(0.157,0.309,0.000), \quad(0.327,0.462$, $0.000)$ and $(0.430,0.393,0.500)$, respectively. S-carbon belongs to the $\mathrm{Cmcm}$ space group and its equilibrium lattice constants from GGA calculations are $\mathrm{a}=2.523 \AA$,

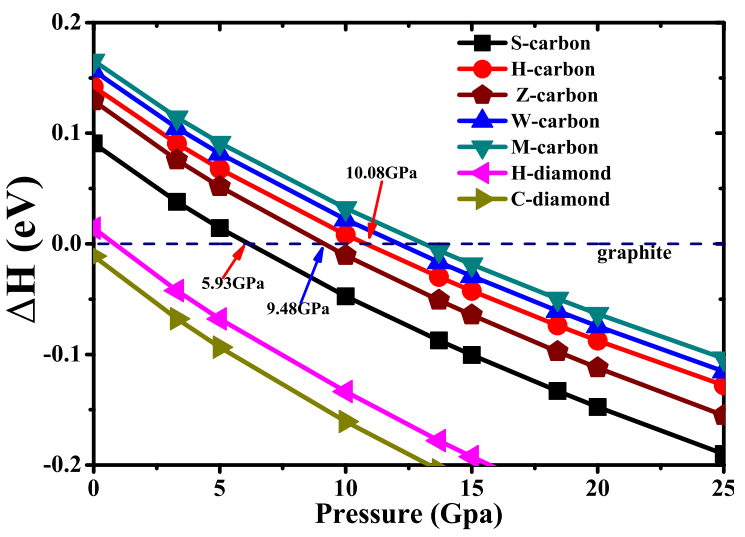

FIG. 2: The enthalpy per atom for cubic diamond, hexagonal diamond, M-carbon, W-carbon, Z-carbon, H-carbon and Scarbon as a function of pressure relative to graphite.

$\mathrm{b}=11.385 \AA$ and $\mathrm{c}=4.899 \AA$. Four inequivalent atoms in S-carbon locate at positions of $(0.500,0.778,0.250)$, $(0.500,0.442,0.078),(0.500,0.132,0.518)$ and $(0.000$, $0.300,0.750)$. Recently proposed Z-carbon contains even number carbon rings and hold Cmmm symmetry, with equilibrium lattice parameters $\mathrm{a}=8.772 \AA, \mathrm{b}=4.256 \AA$ and $\mathrm{c}=2.514 \AA$. There are only two inequivalent atoms in Z-carbon located at $(0.089,0.316,0.500)$ and $(0.167$, $0.185,0.000)$. All the results of the lattice constants of diamond, M-carbon, W-carbon, Z-carbon, H-carbon and S-carbon derived from both GGA and LDA calculations are listed in Tab. I] The LDA calculations tend to give smaller lattice constants for all structures considered here, but the conclusions derived from LDA and GGA are consistent.

The relative stability of diamond, M-carbon, Wcarbon, Z-carbon, H-carbon and S-carbon is evaluated through comparing their cohesive energy per atom. The energy of $\mathrm{H}$-carbon is about $15 \mathrm{meV}$ lower than that of $\mathrm{W}$-carbon, about $25 \mathrm{meV}$ lower than that of M-carbon, and $10 \mathrm{meV}$ higher than that of Z-carbon at both LDA and GGA level. Namely, H-carbon is more stable than $\mathrm{M}$-carbon and W-carbon but less stable than Z-carbon. Among these allotropes, S-carbon is the most stable one whose cohesive energy is about $30 \mathrm{meV}$ lower than that of Z-carbon. From the cohesive energy, S-carbon is the most stable carbon allotrope theoretically proposed until now. The enthalpy per atom for cubic diamond, hexagonal diamond, M-carbon, W-carbon, Z-carbon, $\mathrm{H}$-carbon as well as S-carbon as a function of pressure relative to graphite derived from LDA calculations are shown in Fig. 22 The results indicate that H-carbon is more stable than graphite when the external pressure is larger than $10.08 \mathrm{GPa}$. H-carbon is always favorable than $\mathrm{M}$-carbon and $\mathrm{W}$-carbon at the pressure range from 0 to $25 \mathrm{GPa}$. Amazingly, the transition pressure point of S-carbon is only $5.93 \mathrm{Gpa}$, which is smaller than 
TABLE I: Space group, exchange-correlation functional (EXC), lattice parameters (LP), density $\left(\mathrm{g} / \mathrm{cm}^{3}\right)$, band gap (Eg: eV), cohesive energy (Ecoh: eV) and bulk modulus (B0: Gpa) for diamond, M-carbon, W-carbon, Z-carbon, H-carbon and S-carbon.

\begin{tabular}{|c|c|c|c|c|c|c|c|}
\hline Systems & Space group & EXC & LP & Density & $\mathrm{Eg}$ & Ecoh & B0 \\
\hline diamond & $\mathrm{Fd}-3 \mathrm{~m}$ & LDA & $\mathrm{a}=\mathrm{b}=\mathrm{c}=3.536 \AA$ & 3.611 & 4.648 & -8.997 & 514.08 \\
\hline diamond & $\mathrm{Fd}-3 \mathrm{~m}$ & GGA & $\mathrm{a}=\mathrm{b}=\mathrm{c}=3.574 \AA$ & 191 & 4.635 & -7.693 & 478.96 \\
\hline M-carbon & $\mathrm{C} 2 / \mathrm{m}$ & LDA & $\mathrm{a}=9.093 \AA, \mathrm{b}=2.498 \AA, \mathrm{c}=4.108 \AA$, beta $=96.96^{\circ}$ & 3.443 & 3.532 & -8.821 & 447.33 \\
\hline M-carbon & $\mathrm{C} 2 / \mathrm{m}$ & GGA & $\mathrm{a}=9.194 \AA, \mathrm{b}=2.525 \AA, \mathrm{c}=4.151 \AA$, beta $=97.03^{\circ}$ & 3.333 & 3.493 & -7.531 & 431.44 \\
\hline W-carbon & Pnma & LDA & $\mathrm{a}=8.984 \AA, \mathrm{b}=4.116 \AA, \mathrm{c}=2.498 \AA$ & & 4.325 & -8.830 & 468.12 \\
\hline W-carbon & Pnma & GGA & $\mathrm{a}=9.086 \AA, \mathrm{b}=4.156 \AA, \mathrm{c}=2.525 \AA$ & & 4.281 & -7.539 & 447.03 \\
\hline Z-carbon & $\mathrm{Cmmm}$ & LDA & $\mathrm{a}=8.677 \AA, \mathrm{b}=4.211 \AA, \mathrm{c}=2.489 \AA$ & 07 & 3.414 & -8.857 & 497.87 \\
\hline Z-ca & $\mathrm{Cmmm}$ & GGA & $\mathrm{a}=8.772 \AA, \mathrm{b}=4.256 \AA, \mathrm{c}=2.514 \AA$ & 3.394 & 3.273 & -7.564 & 464.13 \\
\hline $\mathrm{H}-\mathrm{ca}$ & Pbam & LDA & $\mathrm{a}=7.792 \AA, \mathrm{b}=4.757 \AA, \mathrm{c}=2.497 \AA$ & 3.440 & 4.512 & -8.845 & 466.92 \\
\hline H-carbon & Pbam & GGA & $\mathrm{a}=7.874 \AA, \mathrm{b}=4.807 \AA, \mathrm{c}=2.524 \AA$ & 3.339 & 4.459 & -7.554 & 445.88 \\
\hline S-carbon & & LDA & $\mathrm{a}=2.496 \AA, \mathrm{b}=11.293 \AA, \mathrm{c}=4.857 \AA$ & 3.489 & 4.451 & -8.896 & 486.29 \\
\hline S-carbon & $\mathrm{Cmcm}$ & GGA & $\mathrm{a}=2.523 \AA, \mathrm{b}=11.385 \AA, \mathrm{c}=4.899 \AA$ & 3.399 & 4.342 & -7.593 & 468.45 \\
\hline
\end{tabular}

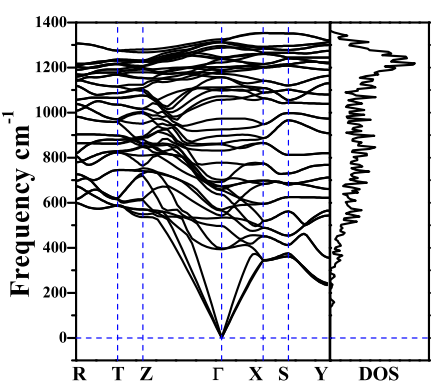

(a)

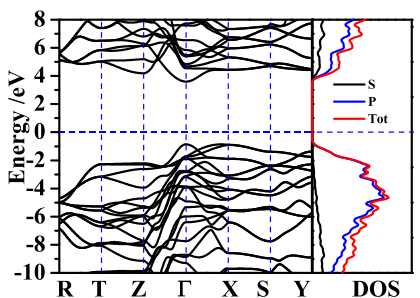

(c)

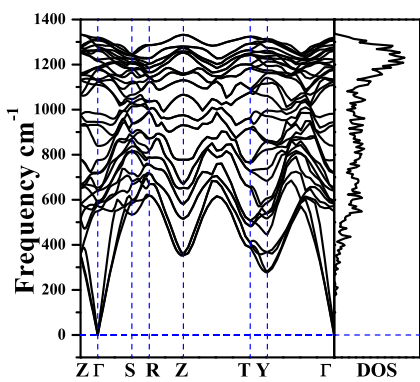

(b)

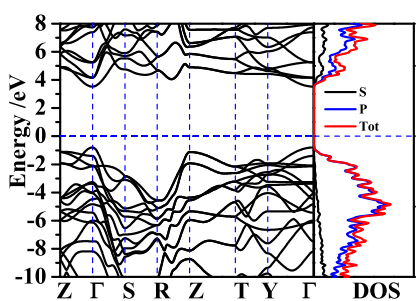

(d)
FIG. 3: Phonon band structure and density of state for Hcarbon (a) and S-carbon (b). Electronic band structure and density of state for H-carbon (c) and S-carbon (d).

all of the previously proposed carbon allotropes. To further confirm the dynamic stabilities of H-carbon and S-carbon, we calculate their phonon band structures and phonon densities of state as shown in Fig. 3. Both LDA and GGA calculations indicate that there is no negative frequency for both structures confirming that H-carbon and S-carbon are dynamic stable phases of carbon.

Space group, density, band gap, cohesive energy and bulk modulus of diamond, M-carbon, W-carbon, Z-carbon, H-carbon, and S-carbon are also summarized in Tab. I] The results indicate that, as superhard intermediate phases between graphite and diamond, M-carbon, W-carbon, Z-carbon, H-carbon, and S-carbon can be formed by cold compressing graphite and their densities and bulk modulus are close to that of diamond. The density of $\mathrm{H}$-carbon and S-carbon derived from GGA is $3.339 \mathrm{~g} / \mathrm{cm}^{3}$ and $3.399 \mathrm{~g} / \mathrm{cm}^{3}$, respectively, which is similar to that of M-carbon $\left(3.333 \mathrm{~g} / \mathrm{cm}^{3}\right)$, W-carbon $\left(3.345 \mathrm{~g} / \mathrm{cm}^{3}\right)$ and Z-carbon $\left(3.394 \mathrm{~g} / \mathrm{cm}^{3}\right)$ derived from the same method. The bulk modulus of $\mathrm{H}$-carbon is $445.88 \mathrm{GPa}$ which is almost the same as that of W-carbon (447.03 GPa) and slightly larger than that of M-carbon (431.44 GPa). The bulk modulus of $\mathrm{S}$-carbon is $468.45 \mathrm{Gpa}$ which is close to that of Z-carbon (466.13 Gpa). The values of the bulk modulus indicate that $\mathrm{H}$-carbon and S-carbon are superhard materials which are comparable to diamond (478.96 Gpa).

Electronic properties of diamond, M-carbon, Wcarbon, Z-carbon, H-carbon and S-carbon are also investigated under both GGA and LDA calculations. All these superhard carbon allotropes are indirect wide band gap semiconductors except for S-carbon. The GGA results show that $\mathrm{H}$-carbon holds an indirect wide gap of $4.531 \mathrm{eV}$ which is larger than that of M-carbon $(3.523 \mathrm{eV}$ ), W-carbon (4.325 eV ) and Z-carbon (3.273 eV), and smaller than that of diamond $(4.635 \mathrm{eV})$. Different from other previously proposed superhard carbon allotropes, S-carbon is a direct-band-gap semiconductor with a gap of $4.342 \mathrm{eV}$. The wide band gap indicates that both $\mathrm{H}$ carbon and S-carbon are transparent carbon allotropes. Fig. 3(c) and (d) show the electronic band structures and densities of states of $\mathrm{H}$-carbon and S-carbon derived from GGA. The densities of states indicate that the valence band maximum for both $\mathrm{H}$-carbon and S-carbon is mainly contributed from the $2 \mathrm{p}$ states of carbon, whereas the conduction band minimum comes from the sp hybridized states.

The simulated X-ray diffraction (XRD) patterns for graphite, Z-carbon, $\mathrm{H}$-carbon and $\mathrm{S}$-carbon at the pressure of $23.9 \mathrm{Gpa}$ are shown in Fig. 4 to compare 


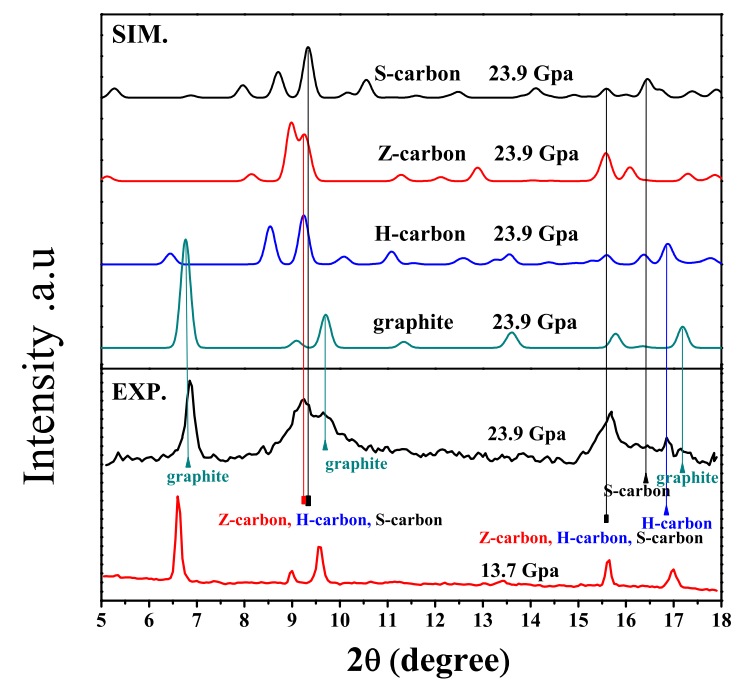

FIG. 4: Simulated XRD data for S-carbon, Z-carbon, Hcarbon, and graphite at corresponding pressure as well as the experimental XRD data at 23.9 Gpa and 13.7 GPa for the superhard intermediate state derived from reference [5].

with the experimental data from reference [5]. The experimental data under high pressure can be explained by $\mathrm{H}$-carbon and S-carbon to some extent. The main $\mathrm{XRD}$ peaks located in the region between $8.5^{\circ}-11^{\circ}$ and $15^{\circ}-17^{\circ}$ for $\mathrm{H}$-carbon and S-carbon agree well with the experimental observations. As indicated by Amsler et al.[11] that sole comparison between experimental and theoretical XRD can not directly clarify the new carbon phase. In view of the cohesive energy, dynamical stability, electronic structure, bulk modulus, lowest phase transition pressure point, and especially the natural transition from $\mathrm{AB}$ stacking graphite, $\mathrm{H}$-carbon and S-carbon are excellent candidates for the new superhard carbon phase.

Note. added During the course of our submission, we became aware of several recent papers [23 -30] reporting the new superhard carbon phases similar to our H-carbon [23, 26] and S-carbon [24, 26]

\section{CONCLUSION}

In summary, we propose two new superhard carbon phases, H-carbon and S-carbon, as the possible candidates for the intermediate phase of cold compressing graphite. Both $\mathrm{H}$-carbon and $\mathrm{S}$-carbon with $\mathrm{sp}^{3}$ carbon bonds are optical transparent superhard carbon phases. They are more stable than M-carbon and W-carbon, and S-carbon is the most table carbon phase theoretically proposed until now. Moreover, when the pressure is above $5.93 \mathrm{Gpa}$ (10.08 Gpa), S-carbon (H-carbon) is more favorable than graphite. These two new members together with the previous proposed M-carbon, bct$\mathrm{C} 4, \mathrm{~W}$-carbon and Z-carbon will enrich the theoretical evidence for understanding the experimental observation.

\section{Acknowledgement}

This work is supported by the National Natural Science Foundation of China (Grant Nos. 11074211, 10874143 and 10974166), the Cultivation Fund of the Key Scientific and Technical Innovation Project, the Program for New Century Excellent Talents in University (Grant No. NCET-10-0169), and the Scientific Research Fund of Hunan Provincial Education Department (Grant Nos. 10K065, 10A118, 09K033)

* Electronic address: lzsun@xtu.edu.cn

$\dagger$ Electronic address: zhong.xtu@gmail.com

[1] F. P. Bundy, J. Chem. Phys. 46, 3437 (1967).

[2] A. F. Goncharov, I. N. Makarenko, and S. M. Stishov, Sov. Phys. JETP 69, 380 (1989).

[3] M. Hanfland, H. Beister, and K. Syassen, Phys. Rev. B 39, 12598 (1989).

[4] W. Utsumi and T. Yagi, Science 252, 1542 (1991).

[5] W. L. Mao, H. Mao, P. J. Eng, T. P. Trainor, M. Newville, C. C. Kao, D. L. Heinz, J. F. Shu, Y. Eng and R. J. Hemley, Science 302, 425 (2003).

[6] A. R. Oganov and C. W. Glass, J. Chem. Phys. 124 244704 (2006).

[7] Q. Li, Y. M. Ma, A. R. Oganov, H. B. Wang, H. Wang, Y. Xu, T. Cui, H. K. Mao, and G. T. Zou, Phys. Rev. Lett. 102, 175506 (2009).

[8] K. Umemoto, R. M. Wentzcovitch, S. Saito, and T. Miyake, Phys. Rev. Lett. 104, 125504 (2010).

[9] J. T. Wang, C. F. Chen and Y. Kawazoe, Phys. Rev. Lett. 106, 075501 (2011).

[10] D. Selli, I. A. Baburin, R. Martoňák and S. Leoni, Phys. Rev. B 84 161411(R) (2011).

[11] M. Amsler, J. A. Flores-Livas, L. Lehtovaara, F. Balima, S. A. Ghasemi, D. Machon, S. Pailhès, A. Willand, D. Caliste, S. Botti, A. S. Miguel, S. Goedecker and M. A. L. Marques, Phys. Rev. Lett. 108065501 (2012).

[12] Z. S. Zhao, B. Xu, X. F. Zhou, L. M. Wang, B. Wen, J. L. He, Z. Y. Liu, H. T. Wang and Y. J. Tian, Phys. Rev. Lett. 107215502 (2011).

[13] R. Z. Khaliullin, H. Eshet, T. D. Küne, J. Behler and M. Parrinello, Nat. Mater. textbf10, 693 (2011).

[14] D. M. Ceperley and B. J. Alder, Phys. Rev. Lett. 45, 566 (1980).

[15] J. P. Perdew and A. Zunger, Phys. Rev. B 23, 5048 (1981).

[16] J. P. Perdew and Y. Wang, Phys. Rev. B 45, 13244 (1992).

[17] G. Kresse and J. Furthmüller, Phys. Rev. B 54, 11169 (1996). 
[18] G. Kresse and J. Furthmüller, Comput. Mater. Sci. 6, 15 (1996).

[19] P. E. Blöchl, Phys. Rev. B 50, 17953 (1994).

[20] G. Kresse and D. Joubert, Phys. Rev. B 59, 1758 (1999).

[21] $13 \times 13 \times 13,5 \times 15 \times 11,5 \times 11 \times 15,5 \times 11 \times 15,5 \times 11 \times 15$ and $15 \times 5 \times 11$ for diamond, M-carbon, W-carbon, Zcarbon, H-carbon and S-carbon, respectively.

[22] K. Parlinski, Z-. Q. Li, and Y. Kawazoe, Phys. Rev. Lett. 78, 4063 (1997).

[23] J. T. Wang, C. F. Chen and Y. Kawazoe, Phys. Rev. B 85, 033410 (2012).

[24] D. Li, K. Bao, F. B. Tian, Z. W. Zeng, Z. He, B. B. Liu and T. Cui, Phys. Chem. Chem. Phys. 14, 4347 (2012).

[25] F. Tian, X. Dong, Z. S. Zhao, J. L. He and H. T. Wang,
J.Phys: Condens. Matter. 24, 165504 (2012).

[26] H. Y. Niu, X. Q. Chen, S. B. Wang, D. Z. Li, W. L. Mao and Y. Y. Li, Phys. Rev. Lett. 108, 135501 (2012).

[27] R. L. Zhou and X. C. Zeng, J. Am. Chem. Soc. 134, 7530 (2012).

[28] M. Amsler, J. A. Flores-Livas, S. Botti, M. A. L Marques and S Geodecker, arXiv:1202.6030v1.

[29] Q. Zhu, Q. Zeng and A. R. Oganov. Phys. Rev. B 85, 201407 (2012).

[30] C. Y. He, L. Z. Sun, C. X. Zhang, X. Y. Peng, K. W. Zhang and J. X. Zhong, Phys. Chem. Chem. Phys. 14, 8410 (2012). 